\title{
A METALLURGICAL APPROACH TO THE CRACKING RESISTANCE OF HOT-DIP GALVANIZED ZINC COATINGS
}

Dedicated to Prof. P. Klimanek on the occasion of his 60th birthday

\author{
S. LAZIK, C. ESLING, M. J. PHILLIPPE*, J. WEGRIA** and M. DUBOIS*** \\ *Laboratoire de Métallurgie des Matériaux Polycristallins (LM2P), E.P. CNRS 98, \\ Université de Metz, 57045 Metz, France \\ **Union Minière (UM), Recherche et Développement, Usine des ASTURIES, \\ 59950 Auby les Douai, France \\ ***Cockerill Sambre (CS), Branche Phenix, Quai du Halange, 10, \\ 4400 Flémalle, Belgique
}

(Received 11 November 1995)

The texture of zinc coatings influences to a large extent their formability, due to the anisotropic properties of zinc. The (0002) basal plane is the easiest slip plane in zinc crystal. Its inclination to the deformation axis and direction directly influences the deformation behaviour. This work deals with the effect of the texture and microstructure on the cracking behaviour of zinc coatings. For this purpose, twelve different commercial hot-dip zinc coatings have been studied.

KEY WORDS: Hot-dip zinc coating, cracking, coating texture, coating microstructure.

\section{INTRODUCTION}

Zinc coated steel sheets are increasingly used, for example in the automobile industry. However, some problems arise in the forming of these materials. One of the problems is the cracking of the zinc coating, which decreases not only the corrosion resistance of the steel sheet but also the life-span of the stamping machines from the production process. Changes in the processing parameters or the addition of alloying elements may be a way to improve the coating characteristics. The addition of $\mathrm{Al}$ to the hot-dip zinc bath, for example, reduces the thickness of the undesirable intermetallic layer and contributes to refining the grains in the coating thus improving its formability.

A great part of the published works concerns the electrogalvanized zinc coatings. Unfortunately, the part of published works about hot-dipped zinc coatings is small [Lazik, Esling, Wegria, 1995]. Besides, the correlation between texture sharpness and cracking behaviour had never been studied. Therefore, we carried out the following experiments in order to study the cracking of hot-dip sheets in relation to both their microstructure and texture. 


\section{EXPERIMENTAL PROCEDURE}

\section{Material}

Twelve hot-dip galvanized steel sheets produced commercially have been investigated. The level of the alloying elements $\mathrm{Al}$ and $\mathrm{Pb}$ can be seen in Table 1 .

Beside the zinc coatings, there are also a Galfan ${ }^{\circledR}$ alloy $(4.7 \% \mathrm{Al}$, sample 12$)$ and a alloy with $1.1 \% \mathrm{Al}$ and low Si content (sample 7). The coating weight, average coating thickness and additionally an indication of wether a skin pass has been carried out or not, are listed in the table.

\section{Experimental Work}

Incomplete (0002) pole figures of the zinc coatings have been measured by $\mathrm{X}$-ray diffraction (Siemens D 5000, 4 circle texture goniometer with Bragg-Brentano setting). The pole figures have been measured up to a tilt angle of $78.5^{\circ}$ (step width: tilt $\Delta \chi=2.5^{\circ}$, azimut $\Delta \varphi=5^{\circ}$ ). The measurement conditions have been kept constant. The pole figure densities have been pseudo-normalized, i.e. exclusively the measured areas $\left(0 \leq \chi \leq 78.5^{\circ}\right)$ have been considered in the normalization procedure.

The coated surfaces of the undeformed samples have been prepared metallographically (mechanical polishing and chemical etching) and observed with an optical microscope (Zeiss, Axiophot). The grain size has been determined by means of linear analysis and will be given in this report approximately.

Table 1 Coating characterization.

\begin{tabular}{lccccc}
\hline Sample & $\begin{array}{c}\text { Coating } \\
\text { weight on } \\
2 \text { faces } \\
{\left[g / m^{2}\right]}\end{array}$ & $\begin{array}{c}\text { average } \\
\text { coating } \\
\text { thickness } \\
{[\mu m]}\end{array}$ & $\begin{array}{c}P b \text { content } \\
{[\%]}\end{array}$ & $\begin{array}{c}\text { Al content } \\
{[\%]}\end{array}$ & Skin pass \\
\hline 01 & 100 & 7 & 0,09 & yes \\
02 & 275 & 19 & 0,12 & no \\
03 & 275 & 19 & $<0,005$ & 0,02 & yes \\
04 & 275 & 19 & $<0,005$ & 0,02 & yes \\
05 & 275 & 19 & 0,07 & no \\
06 & 22 & 18 & & 1,0 & no \\
07 & & 15 & & 1,1 & unknown \\
08 & 275 & 20 & 0,124 & 0,161 & unknown \\
09 & 100 & 7 & 0,124 & 0,165 & no \\
10 & 100 & 14 & 0,124 & 0,165 & yes \\
11 & 255 & 20 & & 4,7 & yes \\
12 & & & & &
\end{tabular}


The cracking behaviour of the zinc layers have been determined by bending tests, carried out in the R\&D centre of Cockerill-Sambre, Belgium.

\section{RESULTS}

\section{Texture}

The samples can be categorized into four groups, according to their (0002) pole figures. A representative example for each group can be seen in Figure 1.

\section{1st Group:}

It can be seen in Figure 1a, that the maximum intensity of the basal planes is located at the pole-figure centre, but the peak shows a broad dispersion and a weak intensity. This indicates that most basal planes are parallel or slightly tilted to the substrate surface, but no strong texture exists. The first group contains samples 03, 07 and 12 .

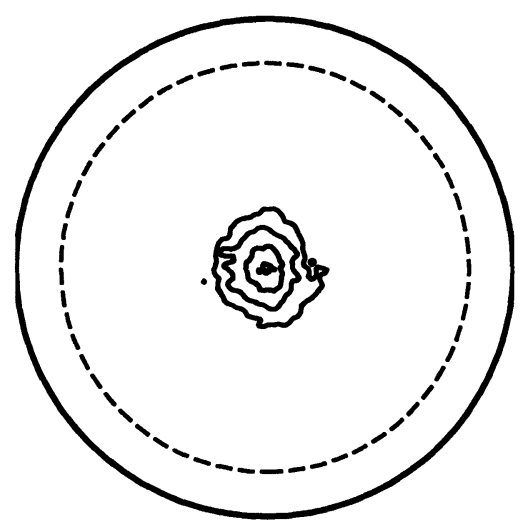

a)

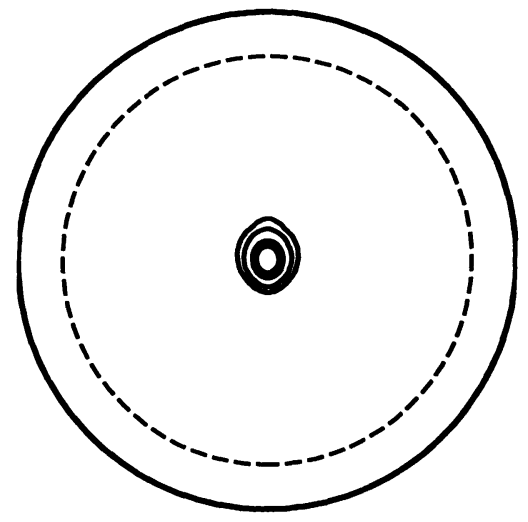

c)

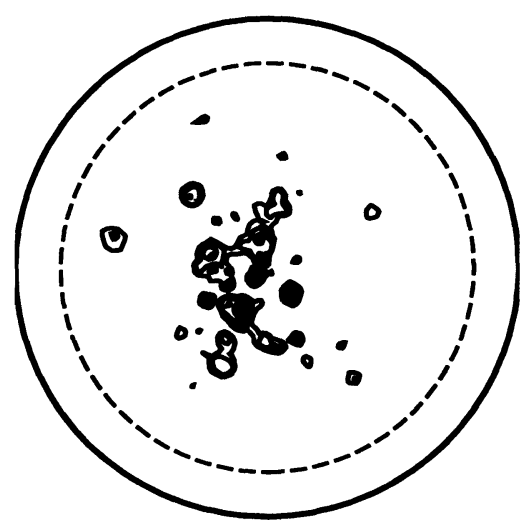

b)

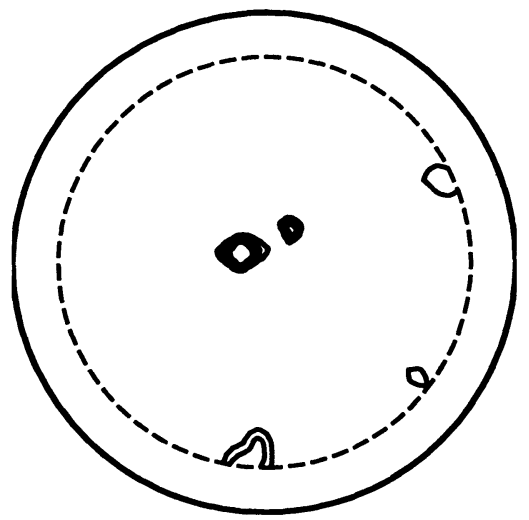

d)

Figure 1 (0002) pole figures of four types of commercial hot-dip coatings, a) sample 07, b) sample 04, c) sample 01 and d) sample 02. 
2nd Group:

Several single peaks are distributed around the pole-figure centre in pole figure $1 \mathrm{~b}$. The maximum intensity is generally located at the pole-figure centre, but separated single orientation peaks appear around the centre even at some distance from it. The maximum intensity is weak or medium as compared to the others, indicating that the texture is not sharp. In addition, little differences in orientation exist inside the grains, as can be inferred from the broadening of the single peaks and metallographical observations. The second group contains samples 04,06 and 08 .

\section{3rd Group:}

The maximum intensity in Figure $1 \mathrm{c}$ is situated in the pole-figure centre, i.e. the basal planes are parallel to the substrate surface. The peak is small and sharp. A strong <0001> fibre texture exists. The third group contains samples $01,05,10$ and 11 .

4th Group:

Several strong single peaks with very high intensities are distributed over the pole figure in Figure 1d. Each single peak in this pole figures can be attributed to the diffraction of one single grain respectively. No reliable statements about the texture are possible due to the poor grain statistics. The fourth group contains samples 02 and 09 .

\section{Microstructure}

Generally, twins have been found in nearly all zinc coatings. However, their aspect may differ, depending on the process conditions and/or alloying elements.

In samples 03 and 04 twins have been found to be very disturbed. The parts of former twins within a same grain do not always remain exactly parallel. The microstructure of sample 03, for example, can be seen in Figure 2. The grain boundaries are locally very disturbed, especially in this sample. Furthermore first recrystallized grains exist already in both samples.

Less disturbed twins have been found in samples $01,05,06,07$ and in sample 02. The microstructure of sample 01 (Figure 3) is similar to that of sample 05. However, an extensive intermetallic layer between the zinc coating and the steel substrate exists in sample 05. Recrystallized grains exist to a large extent in sample 06. Figure 4 shows the microstructure of this coating at low magnification. Large grains with twins can be seen, in which small recrystallized grains without twins are seen. Because of the polarized light used, the various grey nuances are originated by different orientations. In the coating of sample 07 first eutectic precipitations can be found, especially at grain boundaries.

The microstructure of the nearly eutectic coating of sample 12 is characterized by a two-phase alloy, the zinc-rich proeutectic phase being surrounded by an eutectic phase (Figure 5). The eutectic phase consists of alpha aluminium and zinc-rich eta-phase lamellae. The grains appear like rosettes and are divided into many smaller grains or colonies. The dentritic structure of the proeutectic zinc (white) can be observed in the grains.

Samples 08, 09, 10 and 11 show similarities in their microstructures. Clearly visible twins have not been found. However, subgrains were observed in samples 08, 09 and 11 (e.g. sample 08 in Figure 6). The microstructure of sample 10 is more homogeneous than that of sample 11 (type 10 with skin pass), but an extensive intermetallic layer was found at the interface. 


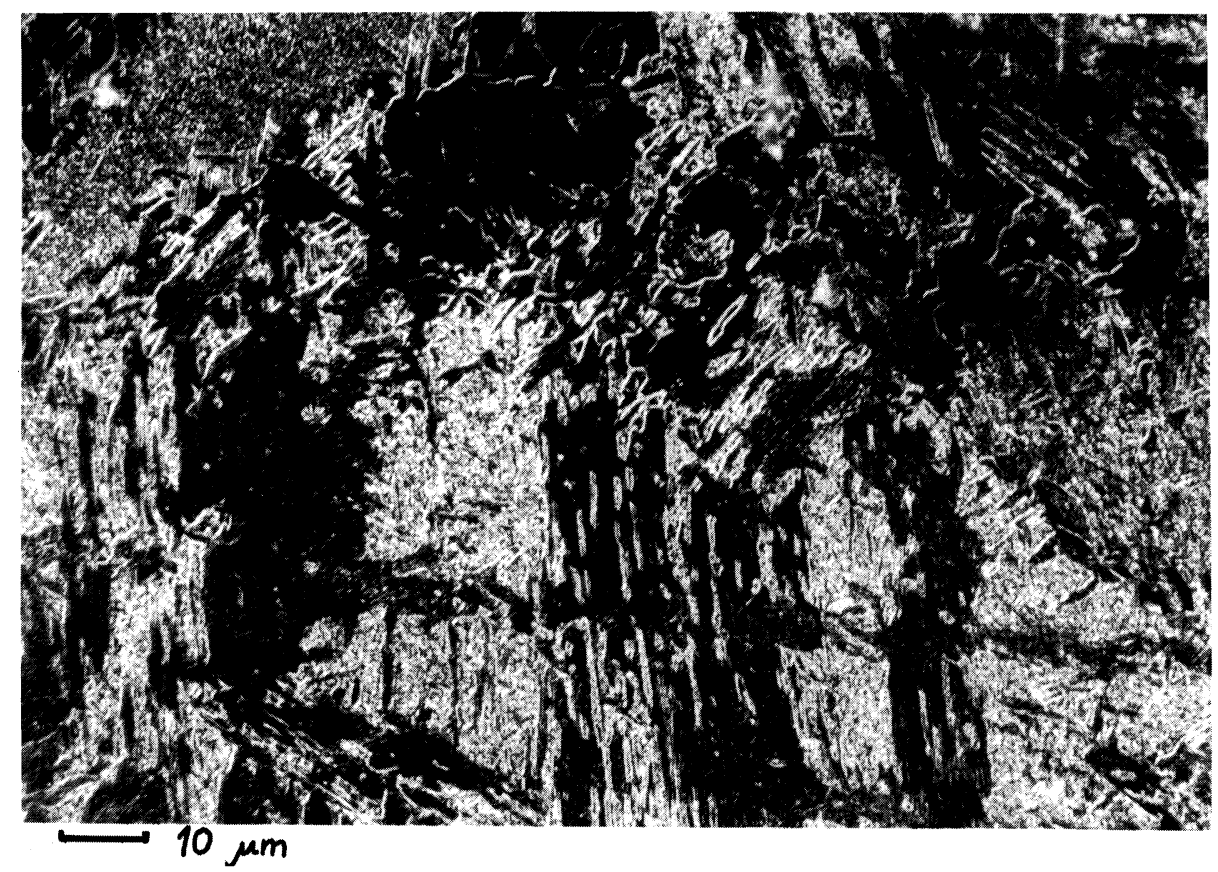

Figure 2 Micrography of sample 03 showing disturbed twins and recrystallized grains. Polarized light.

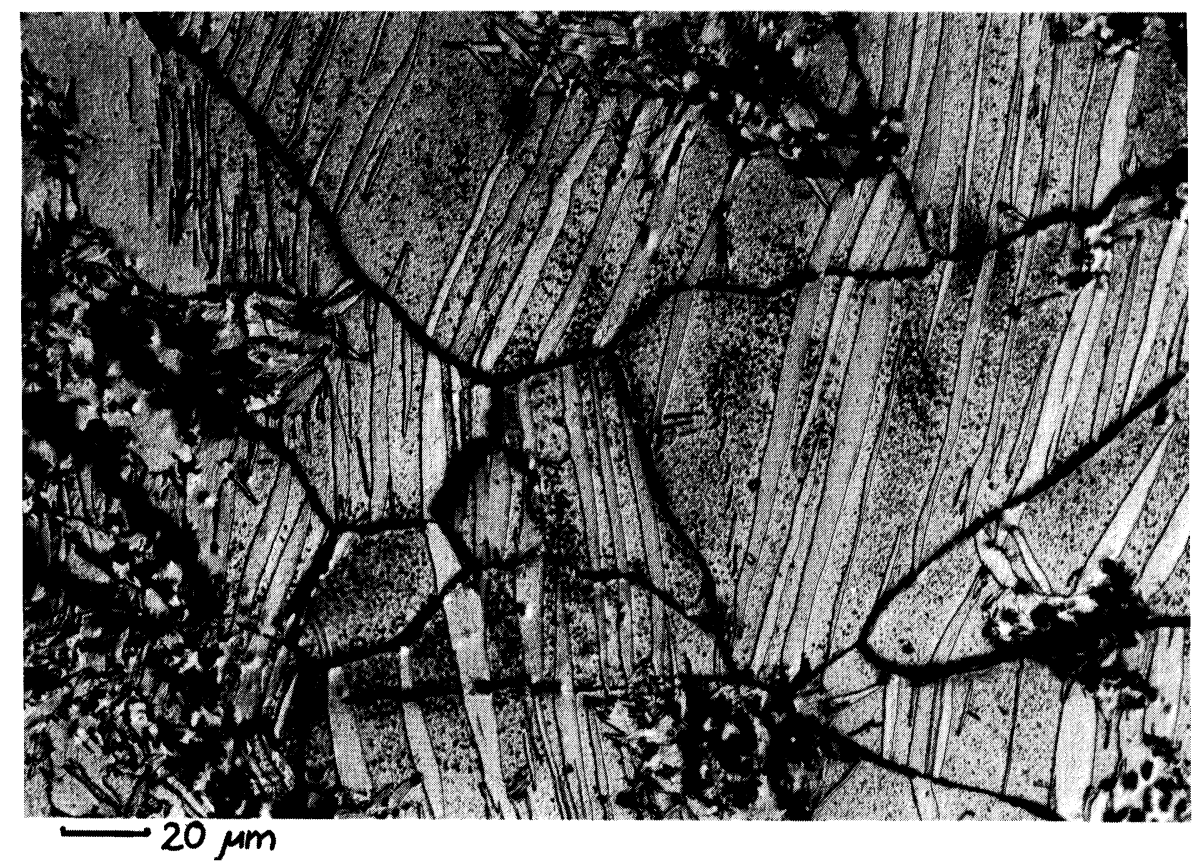

Figure 3 Micrography of sample 01 showing twins in different grains. 


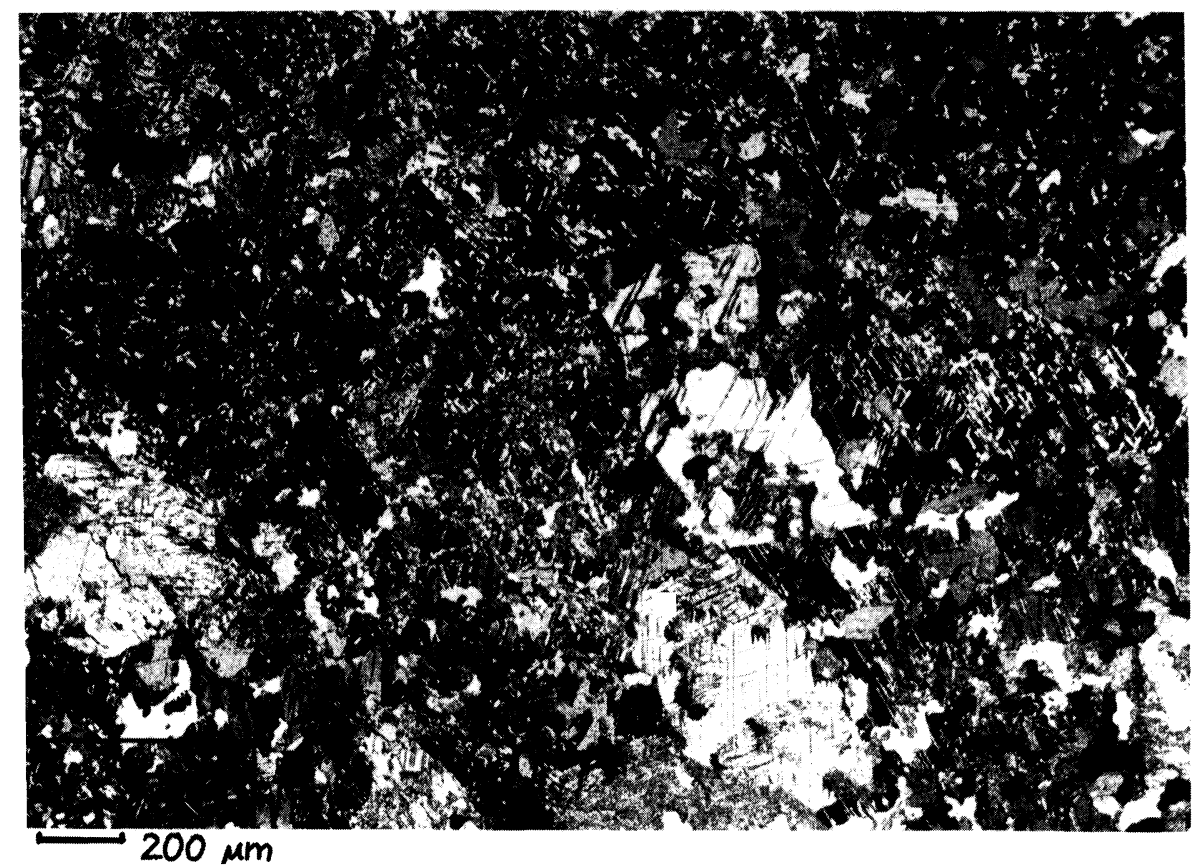

Figure 4 Micrography of sample 06 showing large grains with twins and little recrystallized grains. Polarized light.

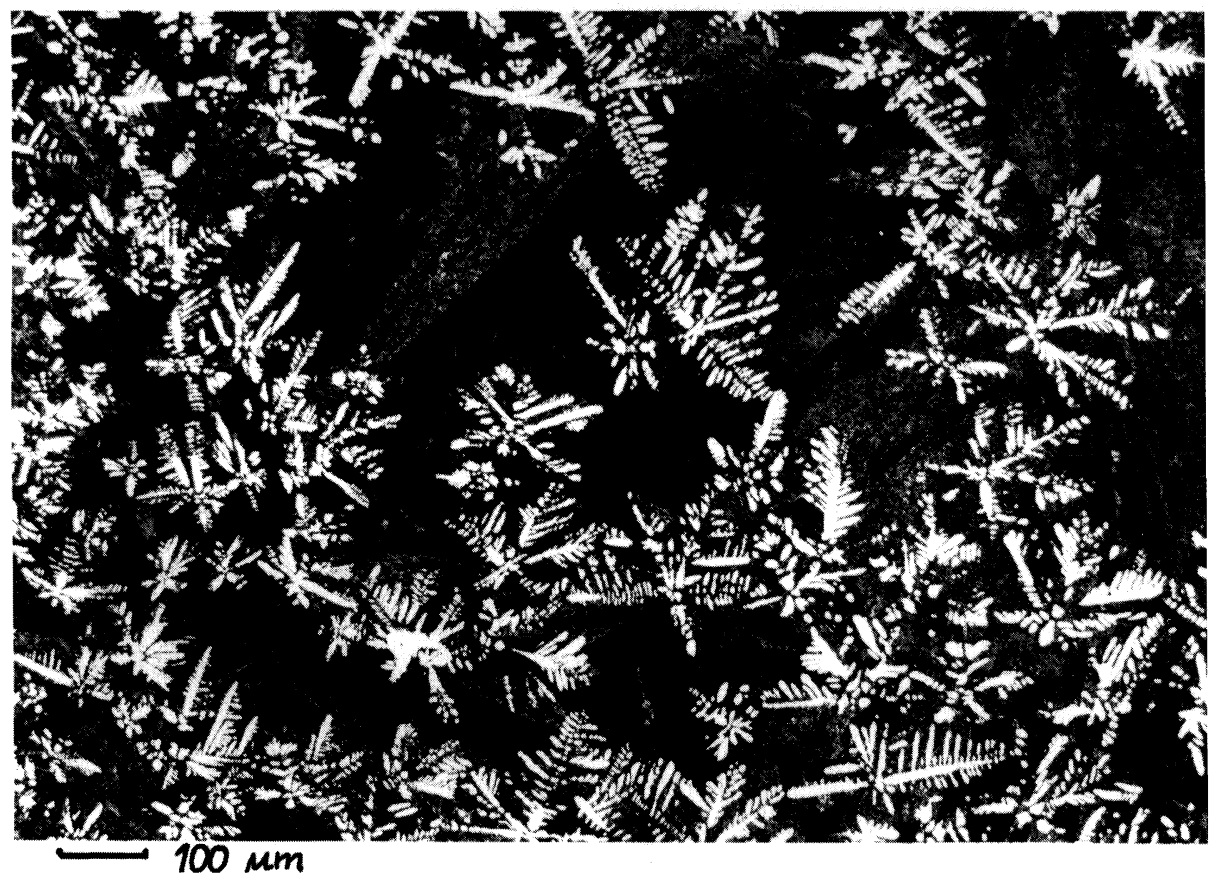

Figure 5 Micrography of sample 12 showing the Galfan alloy, white $\mathrm{Zn}$ dentrites inside the eutectic flowers. Dark field. 


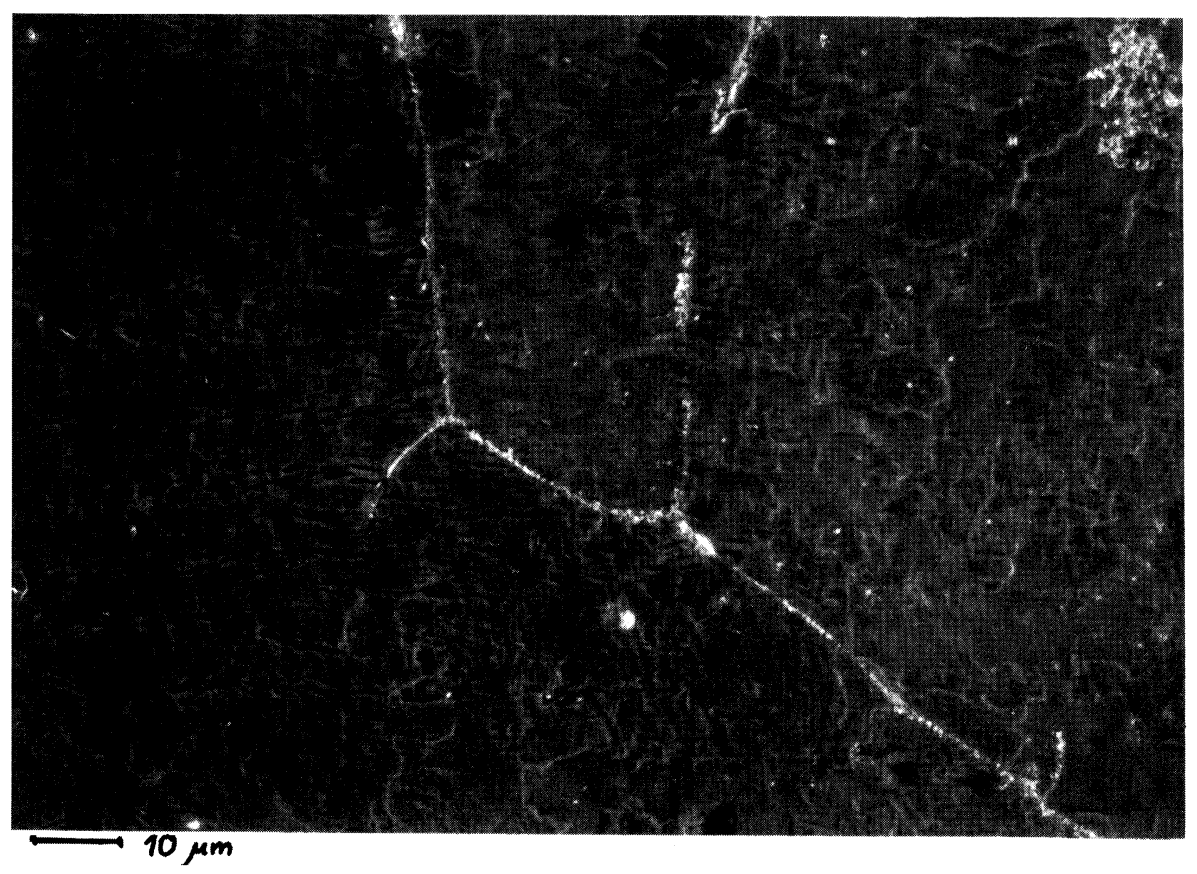

Figure 6 Micrography of sample 08, subgrains into grains. Polarized light.

Intermetallic layers were found in samples $01,02,05$ and 11. A view of such a layer, e.g. in sample 11, after removing the zinc layer, can be seen in Figure 7.

Both the grain size and the cracking behaviour of the samples are summarized with all other results in Table 2 in the discussion.

\section{DISCUSSION}

In Table 2 the samples are ordered according to their cracking resistance, from very high at the top to very low at the bottom of the table. The details about the texture and microstructure of the zinc layers have been gathered in table.

From this table, it can be recognized that the distribution of samples into four different groups according to their pole figures is also significant with respect to their cracking resistance.

Broad and flat peaks but also evenly separated single orientations with weak pole figure densities exist when very good or good cracking behaviour was observed. The broad and weak peaks, in spite of large grains, will be caused by defects such as subgrains and recrystallized grains that may lead to orientation differences inside the grains. The alloy sample 07 without such defects has smaller grains but shows eutectic precipitations.

Medium cracking resistance was found generally for strong $<0001>$ fibre textures. An important difference between these samples and those with good or very good 


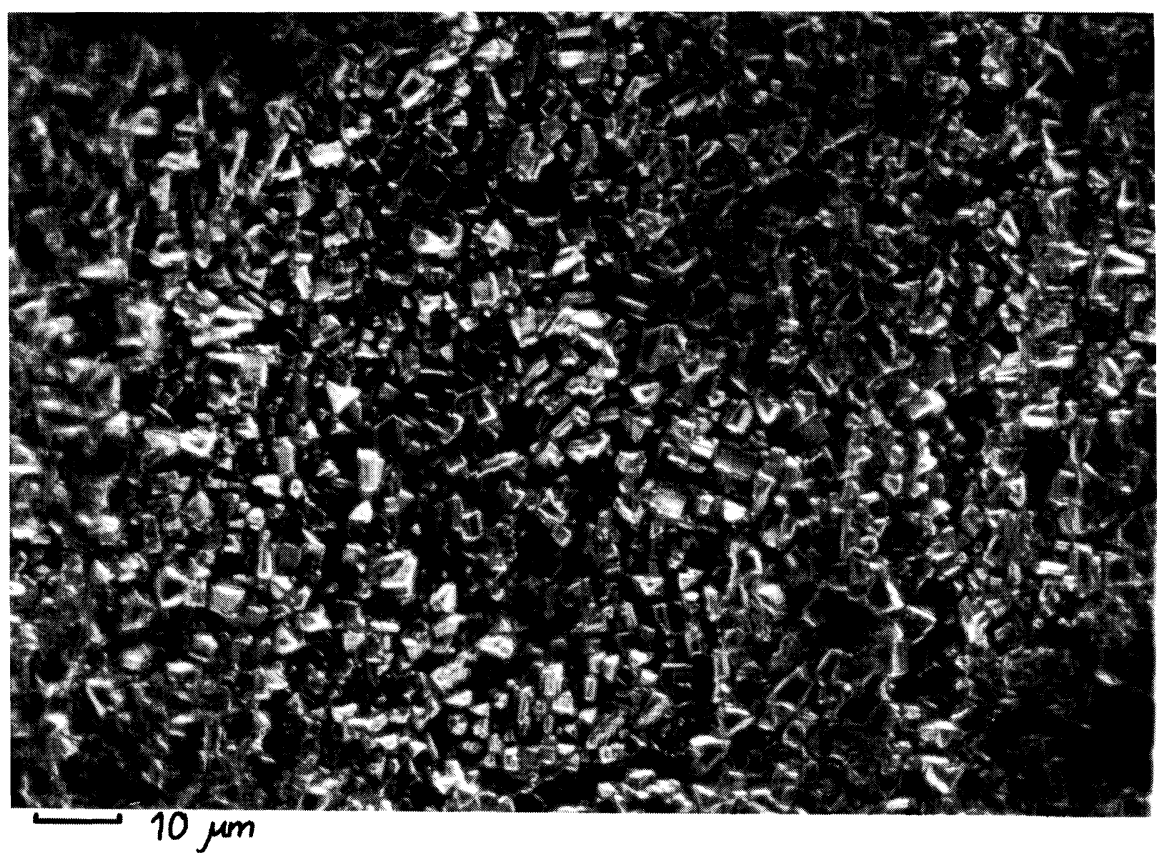

Figure 7 Micrography of the intermetallic layer, sample 11. Dark field.

cracking resistance consists in the existence of less disturbed twins in the first group. Furthermore, an intermetallic layer has been found in the samples 01 and 05 .

Although the pole figure and the microstructure of sample 11 looks similar to sample 10 , low cracking resistance was found. This is probably due to the intermetallic layer, which had not been found in sample 10 .

A brittle layer cannot deform plastically and may induce cracking of the coating during deformation. Particularly, addition of $\mathrm{Al}$ prevents the formation of a thick intermetallic layer.

Very bad cracking resistance exists in samples 02 and 09 with very large grains. Very strong single peaks like single crystal reflexions were measured. An extensive intermetallic layer has been observed in sample 02. Cracking will appear especially in the grains with unfavourable orientation for deformation, i.e. basal planes (0002) parallel or perpendicular to the deformation axis. Therefore, not all the grains will undergo cracking during the deformation, particularly in sample 09 without intermetallic layer.

\section{CONCLUSION}

When considering Table 2, three groups can be distinguished according to their cracking behaviour: very good or good, medium and low or very low cracking resistance.

It can clearly be seen that there is a very good or good cracking resistance when the sharpness of the pole figure is very weak or weak. Likewise, for very high pole figure sharpness very low cracking resistance was found. 
With respect to the grain size, there is no clear direct correlation in the data summarized in Table 2. There are always large grains in the case of very low cracking resistance. But in the case of good or very good cracking resistance, there are both, small and large grains. For example, samples 03 and 07 have very good cracking resistance but the grain sizes differ to a large extent. The grain size of samples 03, 04 and 12 is even larger than in the samples with average cracking behaviour. Therefore, the grain size influences only secondarily the cracking resistance. The differences in cracking resistance will be caused by other factors that have an effect on cracking.

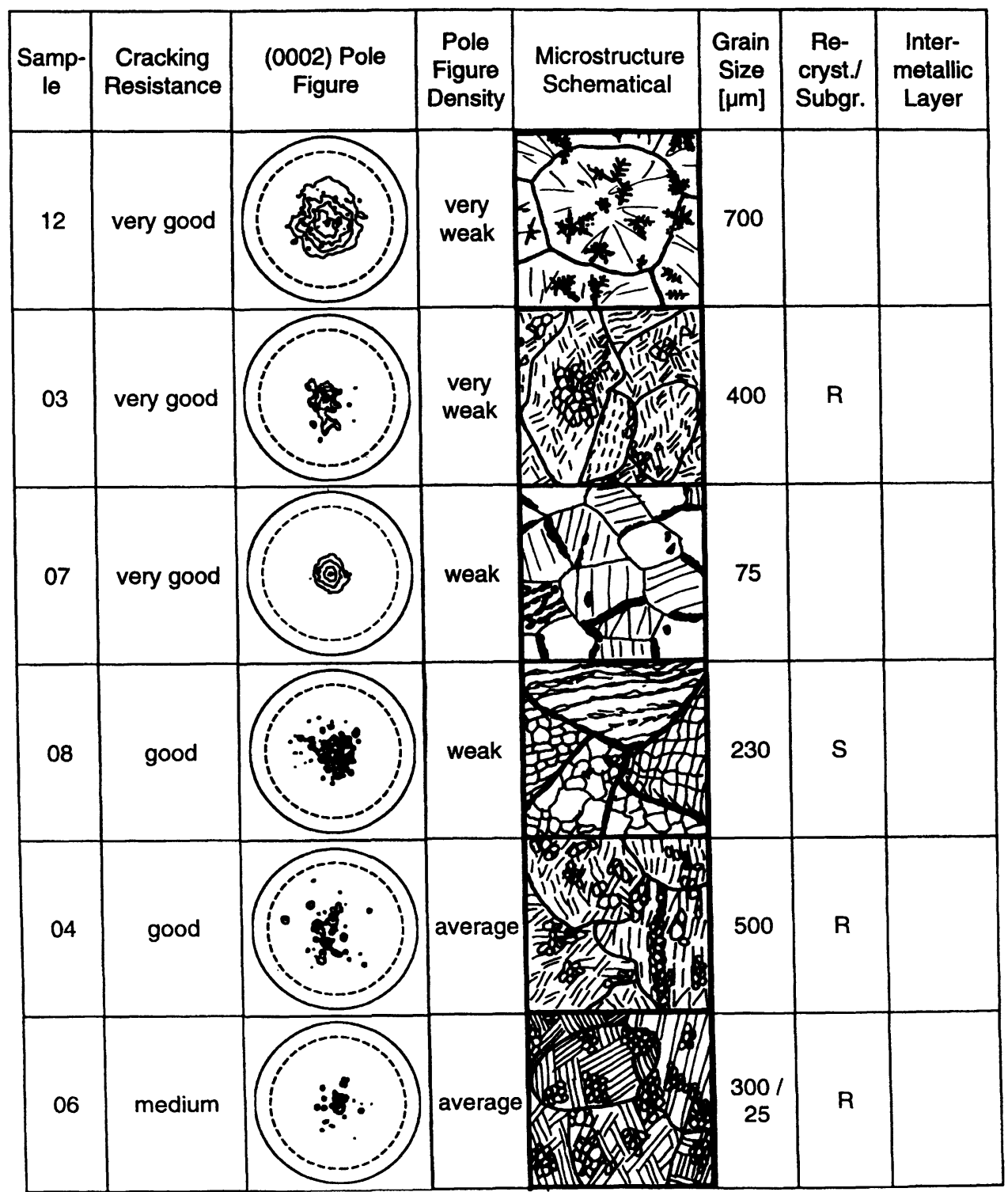

Table 2 Part I 


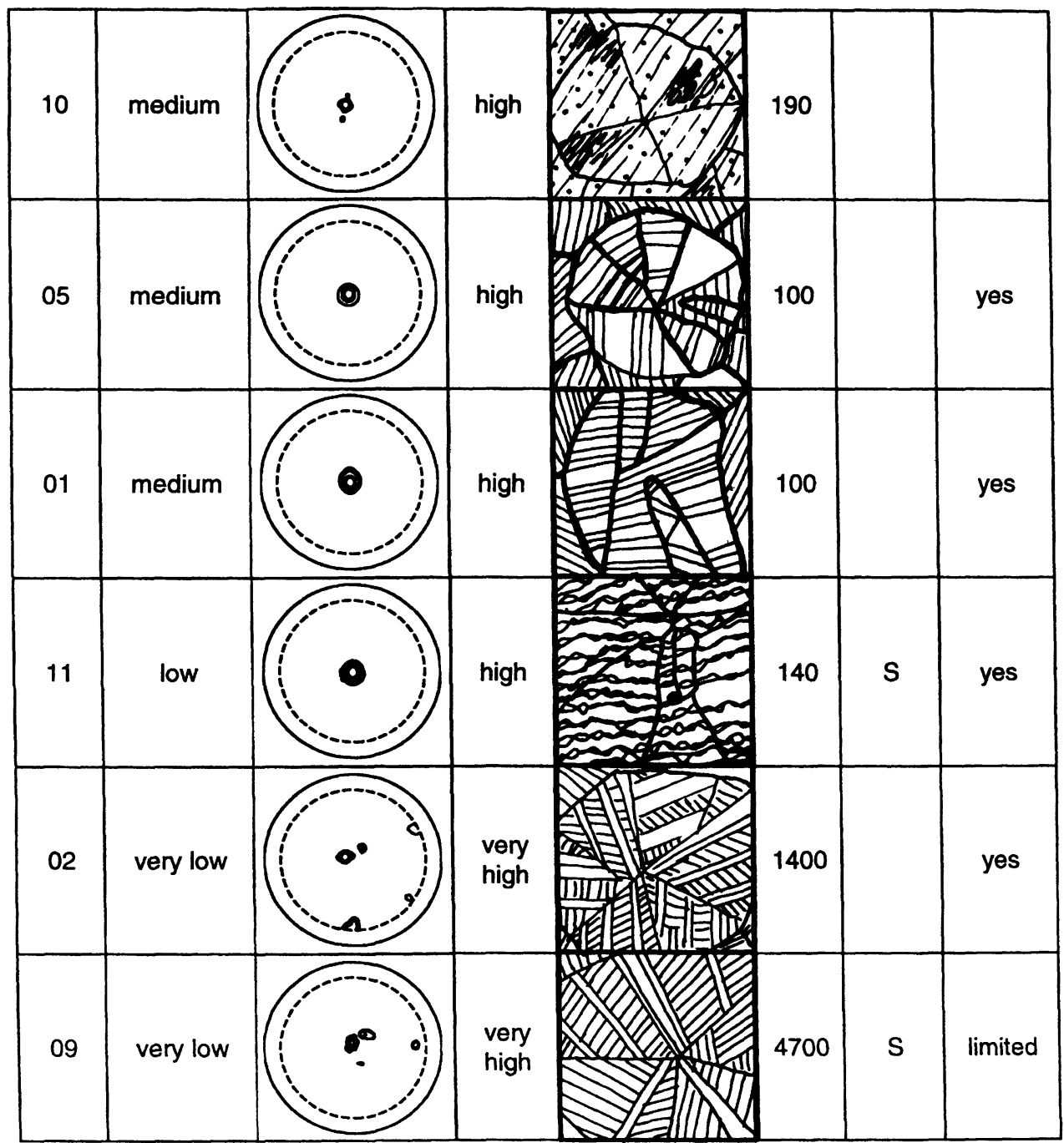

Table 2 Experimental Results (Cracking behaviour, texture and microstructure).

Extensive intermetallic layers were not found in samples with good and very good cracking resistance.

Especially in the samples with good or very good cracking resistance, subgrains or small recrystallized grains have been observed, which may hinder a crack propagation. However, alloys such as Galfan with an eutectic phase have shown to possess also a very good cracking resistance. 


\section{SUMMARY}

A clear correlation was found: the weaker the (0002) pole figure density, the better the cracking resistance of the zinc coating.

Subgrains and recrystallized grains may promote a good cracking resistance.

The grain size has no direct influence on the cracking behaviour of the zinc coating and plays a secondary role.

An extensive intermetallic layer decreases the cracking resistance.

\section{References}

Lazik, S., Esling, C. and Wegria, J. (1995). Cracking in Zinc Layers on Continuous Galvanized SheetsA Review. Textures and Microstruct. 23, 131-147.

Takechi, H., Matsuo, M., Kawasaki, M. and Tamura, T. (1981). Textures and Properties of Metallic Coatings on Sheet Steels. Proc. of ICOTOM 6, S. Nagashima ed., The Iron and Steel Institute of Japan, Tokyo, Japan, 209-222.

Vlad, C. M. (1988). Texture and Corrosion Resistance of Metallic Coatings. In Directional Properties of Materials, H. J. Bunge ed., DGM Informationsgesellschaft mbH. Verlag, Oberursel, Germany, pp 199-212.

Wassermann, G. and Grewen, J. (1962). Texturen metallischer Werkstoffe. Springer Verlag, 2. ed, Berlin/Göttingen/Heidelberg. 